\title{
REMARKS ON THE PARAMETRIZED SYMBOL CALCULUS MICHIO KINOSHITA
}

\begin{abstract}
In his paper, L. Hörmander has used the Weyl calculus to study the Fourier integral operator theory. In the present paper, the author considers the correspondences $W_{\tau}, \tau \in R$ ( $R$ is the set of the real numbers), which mean the standard correspondence of symbol and operator if $\tau=0$, and the correspondence of Weyl type if $\tau=1 / 2$, and shows the explicit asymptotic formula which describes the deviation of $W_{\sigma}\left(W_{\tau}\right)^{-1}$ from the automorphisms as Lie algebra, and makes some remarks on the above formula.
\end{abstract}

\section{Symbol classes.}

NOTATION.

$$
p_{(\beta)}^{(\alpha)}(x, \xi)=\partial_{\xi}^{\alpha} D_{x}^{\beta} p(x, \xi),
$$

where $p(x, \xi) \in C^{\infty}\left(R_{x}^{n} \times R_{\xi}^{n}\right), D_{x_{j}}=-i \partial / \partial x_{j}$,

$$
p_{\left(\beta, \beta^{\prime}\right)}^{\left(\alpha, \alpha^{\prime}\right)}\left(x, \xi, x^{\prime}, \xi^{\prime}\right)=\partial_{\xi}^{\alpha} \partial_{\xi^{\prime}}^{\alpha^{\prime}} D_{x}^{\beta} D_{x^{\prime}}^{\beta^{\prime}} p\left(x, \xi, x^{\prime}, \xi^{\prime}\right),
$$

where $p\left(x, \xi, x^{\prime}, \xi^{\prime}\right) \in C^{\infty}\left(R_{x}^{n} \times R_{\xi}^{n} \times R_{x^{\prime}}^{n} \times R_{\xi^{\prime}}^{n}\right),\langle\xi\rangle=\sqrt{1+|\xi|^{2}},\left\langle\xi ; \xi^{\prime}\right\rangle=$ $\sqrt{1+|\xi|^{2}+\left|\xi^{\prime}\right|^{2}}$ and $\hat{u}(\xi)$ is the Fourier transform of $u(x)$.

We denote by $S_{\rho, \delta}^{m}$, for any real numbers $m, \rho, \delta$ such that $0 \leq \delta \leq \rho \leq 1, \delta<1$, the set of smooth functions $p(x, \xi)$ on $R_{x}^{n} \times R_{\xi}^{n}$ which satisfy the condition that for any multi-indices $\alpha, \beta$, there exists a constant $C_{\alpha, \beta}$ such that

$$
\left|p_{(\beta)}^{(\alpha)}(x, \xi)\right| \leq C_{\alpha, \beta}\langle\xi\rangle^{m+\delta|\beta|-\rho|\alpha|} .
$$

Let $S_{\rho, \delta}^{\infty}$ be the set $\bigcup_{m \in \mathbf{R}} S_{\rho, \delta}^{m}$.

We denote by $S_{\rho, \delta}^{m, m^{\prime}}$, for any real numbers $m, m^{\prime}, \rho, \delta$ such that $0 \leq \delta \leq \rho \leq 1$, $\delta<1$, the set of smooth functions $p\left(x, \xi, x^{\prime}, \xi^{\prime}\right)$ on $R_{x}^{n} \times R_{\xi}^{n} \times R_{x^{\prime}}^{n} \times R_{\xi^{\prime}}^{n}$, which satisfy the condition that for any multi-indices $\alpha, \beta, \alpha^{\prime}, \beta^{\prime}$, there exists a constant $C_{\alpha, \beta, \alpha^{\prime}, \beta^{\prime}}$ such that

$$
\left|p_{\left(\beta, \beta^{\prime}\right)}^{\left(\alpha, \alpha^{\prime}\right)}\left(x, \xi, x^{\prime}, \xi^{\prime}\right)\right| \leq C_{\alpha, \beta, \alpha^{\prime}, \beta^{\prime}}\langle\xi\rangle^{m+\delta|\beta|-\rho|\alpha|}\left\langle\xi ; \xi^{\prime}\right\rangle^{\delta\left|\beta^{\prime}\right|}\langle\xi\rangle^{m^{\prime}-\rho\left|\alpha^{\prime}\right|} .
$$

We denote by $S$ the set of the rapidly decreasing functions, and we denote by $\mathrm{Op}\left(S_{\rho, \delta}^{m}\right)$ the set of pseudo-differential operators which is defined by

$$
(B(p) u)(x)=\int e^{i x \cdot \xi} p(x, \xi) \hat{u}(\xi) d \xi, \quad p \in S_{\rho, \delta}^{m}, u \in \mathcal{S} .
$$

In like manner, we denote by $\operatorname{Op}\left(S_{\rho, \delta}^{m, m^{\prime}}\right)$ the set of pseudo-differential operators

Received by the editors September 13, 1983 and, in revised form, October 19, 1983

1980 Mathematics Subject Classification. Primary 47G05.

Key words and phrases. Pseudo-differential operator. 
which is defined by

$$
\begin{aligned}
(W(p) u)(x)=\iiint e^{i\left(x-x^{\prime}\right) \cdot \xi+i x^{\prime} \cdot \xi^{\prime}} p\left(x, \xi, x^{\prime}, \xi^{\prime}\right) \hat{u}\left(\xi^{\prime}\right) d \xi d x^{\prime} d \xi^{\prime} & \\
& p \in S_{\rho, \delta}^{m, m^{\prime}}, u \in S .
\end{aligned}
$$

We denote by $R_{\tau}^{m}$, for any real numbers $m$ and $\tau$, the linear mapping from $S_{\rho, \delta}^{m}$ to $S_{\rho, \delta}^{m, 0}$, defined by

$$
\left(R_{\tau}^{m}(p)\right)\left(x, \xi, x^{\prime}, \xi^{\prime}\right)=p\left((1-\tau) x+\tau x^{\prime}, \xi\right),
$$

and we denote by $W_{\tau}^{m}$, for any real numbers $m$ and $\tau$, the linear mapping from $S_{\rho, \delta}^{m}$ to $\operatorname{Op}\left(S_{\rho, \delta}^{m}\right)$, defined by $W R_{\tau}^{m}$.

REMARK 1. Op $\left(S_{\rho, \delta}^{m, 0}\right)=\mathrm{Op}\left(S_{\rho, \delta}^{m}\right)$.

REMARK 2. The standard correspondence between symbols and operators is $W_{0}^{m}$, and the correspondence of Weyl type is $W_{1 / 2}^{m}$ (for the Weyl calculus, see [1]).

We denote by $W_{\tau}^{\infty}$, for any real numbers $\tau$, the linear mapping from $S_{\rho, \delta}^{m}$ to $\operatorname{Op}\left(S_{\rho, \delta}^{\infty}\right)$, defined by $W_{\tau}^{\infty}(p)=W_{\tau}^{m}(p)$, for any $p \in S_{\rho, \delta}^{m}$.

Let $A=W_{\tau}^{m}(p)$ and $B=W_{\tau}^{m^{\prime}}(q)$, where $p \in S_{\rho, \delta}^{m}, q \in S_{\rho, \delta}^{m^{\prime}}$. The product $A B$ is contained in $\operatorname{Op}\left(S_{\rho, \delta}^{m+m^{\prime}}\right)$. Therefore, there exists a function $r \in S_{\rho, \delta}^{m+m^{\prime}}$ such that $A B=W_{\tau}^{m+m^{\prime}}(r)$. This symbol $r$ is expressed by the formula

$$
r(x, \xi)=\left[\left(\operatorname{Exp} i\left((1-\tau) D_{\xi} D_{y}-\tau D_{x} D_{\eta}\right)\right) p(x, \xi) q(y, \eta)\right]_{(x, \xi)=(y, \eta)} .
$$

We use the notation $r=p \circ_{\tau} q$ in the following section. This notation is used in [3].

2. Main result. In this section we examine the linear transformation of $\operatorname{Op}\left(S_{\rho, \delta}^{m}\right)$ given by $K_{\sigma, \tau}^{m}=W_{\sigma}^{m}\left(W_{\tau}^{m}\right)^{-1}$, when $\sigma \neq \tau$.

REMARK 3. The fact that $K_{\sigma, \tau}^{\infty}$ is not an automorphism of $\operatorname{Op}\left(S_{\rho, \delta}^{\infty}\right)$ as algebra is reduced to the fact that $a \circ_{\sigma} b \neq a \circ_{\tau} b$ by the composition formula above.

In this section, we consider the deviation of $K_{\sigma, \tau}^{m}$ from the automorphisms of $\operatorname{Op}\left(S_{\rho, \delta}^{m}\right)$ as a Lie algebra.

REMARK 4. When $m \leq \rho-\delta, \mathrm{Op}\left(S_{\rho, \delta}^{m}\right)$ is a Lie algebra. By a trivial computation, we obtain the following fact. When $A \in \mathrm{Op}\left(S_{\rho, \delta}^{m}\right)$ and $B \in \mathrm{Op}\left(S_{\rho, \delta}^{m}\right)$, we get

$$
\left[K_{\sigma, \tau}^{m}(A), K_{\sigma, \tau}^{m}(B)\right]-K_{\sigma, \tau}^{m}([A, B])=W_{\sigma}^{2 m}\left(a \circ_{\sigma} b-b \circ_{\sigma} a-a \circ_{\tau} b+b \circ_{\tau} a\right),
$$

where $a=\left(W_{\tau}^{m}\right)^{-1} A, b=\left(W_{\tau}^{m}\right)^{-1} B$.

We denote by $H_{n}(\sigma)$ the function $\sum_{k=1}^{n} F^{n-k} G^{k-1}$, where $F=(1-\sigma) \xi \cdot y-\sigma x \eta$, $G=(1-\sigma) x \cdot \eta-\sigma \xi \cdot y$.

REMARK 5. This function has an invariant property with respect to the changing of $\xi \cdot y$ and $x \cdot \eta$. Obvious calculation gives

$$
\begin{array}{r}
H_{n}(\sigma)=\sum_{k=1}^{n} \sum_{r=0}^{n-k} \sum_{s=0}^{k-1}(-1)^{n-k-r+s}\left(\begin{array}{c}
n-k \\
r
\end{array}\right)\left(\begin{array}{c}
k-1 \\
s
\end{array}\right) \\
\cdot(1-\sigma)^{k+r-s-1} \sigma^{n+s-k-r}(\xi \cdot y)^{r+s}(x \cdot \eta)^{n-(r+s)-1} .
\end{array}
$$

We denote by $T_{k}(D)$ the operator corresponding to

$$
T_{k}(x, \xi, y, \eta)=\frac{i^{k}}{k !}(\xi y-x \eta)\left(H_{k}(\sigma)-H_{k}(\tau)\right) .
$$

The deviation is described by the following theorem. 
THEOREM. If $a \in S_{\rho, \delta}^{m_{1}}$ and $b \in S_{\rho, \delta}^{m_{2}}$, then

$$
\begin{gathered}
a \circ_{\sigma} b-b \circ_{\sigma} a-a \circ_{\tau} b+b \circ_{\tau} a-\left.\sum_{k=0}^{n} T_{k}(D) a(x, \xi) b(y, \eta)\right|_{(x, \xi)=(y, \eta)} \\
\in S_{\rho, \delta}^{m_{1}+m_{2}-(n+1)(\rho-\delta)} .
\end{gathered}
$$

In the case of $k=0,1,2,3$, we have

$$
\begin{gathered}
T_{0}(x, \xi, y, \eta)=0, \quad T_{1}(x, \xi, y, \eta)=0, \\
T_{2}(x, \xi, y, \eta)=(\sigma-\tau)(\xi y-x \eta)(\xi y+x \eta), \\
T_{3}(x, \xi, y, \eta)=-\frac{i}{2}(\sigma-\tau)(\xi y-x \eta)(\sigma+\tau-1)(\xi y+x \eta)^{2} .
\end{gathered}
$$

PROOF. Essentially the product formula and calculations give the proof.

REMARK 6. $T_{k}(x, \xi, y, \eta)$ is divisible by $(\sigma-\tau)(\xi y-x \eta)$. Consequently

$$
T_{k}(x, \xi, y, \eta)=(\sigma-\tau)(\xi y-x \eta) U_{k}(x, \xi, y, \eta, \sigma, \tau),
$$

where $U_{k}$ is a symmetric function of $\xi \cdot y$ and $x \cdot \eta$, and also a symmetric function of $\sigma$ and $\tau$. By the fact that $T_{2} \neq 0$, we obtain that $K_{\sigma, \tau}^{m}, m \leq \rho-\delta$, is not an automorphism of the Lie algebra $\mathrm{Op}\left(S_{\rho, \delta}^{m}\right)$. By the fact that $T_{0}=T_{1}=0$, we obtain that $\tilde{K}_{\sigma, \tau}^{m}, m \leq \rho-\delta$, which is induced by $K_{\sigma, \tau}^{m}, m \leq \rho-\delta$, is an automorphism of the Lie algebra $\operatorname{Op}\left(S_{\rho, \delta}^{m}\right) / \mathrm{Op}\left(S_{\rho, \delta}^{2 m-2(\rho-\delta)}\right)$.

REMARK 7. From the property that $H_{k}(1-\sigma)=(-1)^{k-1} H_{k}(\sigma)$, we obtain that if $\sigma+\tau=1$ and $k$ is odd, then $T_{k}(x, \xi, y, \eta)=0$.

\section{REFERENCES}

1. L. Hörmander, The Weyl calculus of pseudo-differential operators, Comm. Pure Appl. Math. 32 (1979), 359-443.

2. H. Kumano-go, Gibibun-Sayoso, Iwanami, 1974.

3. M. V. Karasev and V. E. Nazaikinskii, On the quantization of rapidly oscillating symbols, Math. USSR-Sb. 34 (1978), 737-764.

4. I. A. Seresevskii, Quantization in contangent bundles, Soviet Math. Dokl. 20 (1979), 402-405.

Department of Mathematics, Shimane University, Nishi-Kawatsu 1060, MatSUE, JAPAN 690 